\title{
A Small-molecule Kinase Inhibitor, CEP-1347, Inhibits Survivin Expression and Sensitizes Ovarian Cancer Stem Cells to Paclitaxel
}

\author{
KEITA TOGASHI ${ }^{1,2}$, MASASHI OKADA ${ }^{1}$, MASAHIRO YAMAMOTO ${ }^{1}$, SHUHEI SUZUKI ${ }^{1,3}$, \\ TOMOMI SANOMACHI ${ }^{1,3}$, SHIZUKA SEINO ${ }^{1}$, HIDETOSHI YAMASHITA ${ }^{2}$ and CHIFUMI KITANAKA ${ }^{1,4}$ \\ Departments of ${ }^{1}$ Molecular Cancer Science, ${ }^{2}$ Ophthalmology and ${ }^{3}$ Clinical Oncology, \\ Yamagata University School of Medicine, Yamagata, Japan; \\ ${ }^{4}$ Research Institute for Promotion of Medical Sciences, Yamagata University Faculty of Medicine, Yamagata, Japan
}

\begin{abstract}
Background: Chemoresistance of cancer stem cells (CSCs) is considered a major cause of post-treatment recurrence that negatively impacts the prognosis of patients with ovarian cancer. Materials and Methods: Using CSCs derived from two different ovarian cancer cell lines, we searched for molecules implicated in the chemoresistance of ovarian CSCs and also drugs with which to target those molecules. Results: Knockdown of survivin overexpressed in ovarian CSCs resulted in increased sensitivity to paclitaxel. Treatment at clinically relevant concentrations with CEP-1347, a mixed lineage kinase inhibitor with a known safety profile in humans, reduced survivin expression in ovarian CSCs and sensitized them to paclitaxel. Conclusion: Survivin overexpression plays a key role in the chemoresistance of ovarian CSCs. Introduction of CEP1347, which targets survivin expression in ovarian CSCs, as a chemosensitizer for conventional ovarian cancer chemotherapy may serve as a rational and feasible approach for better management of ovarian cancer.
\end{abstract}

Ovarian cancer is one of the most common and lethal gynecological malignancies, being the eighth leading cause of cancer death in women worldwide (1). The standard initial management of ovarian cancer involves maximal cytoreductive, debulking surgery followed by chemotherapy consisting of platinum and taxane (2). Although ovarian cancers are chemosensitive in general, recurrence is

Correspondence to: Chifumi Kitanaka and Masashi Okada, Department of Molecular Cancer Science, Yamagata University School of Medicine, Yamagata, 990-9585, Japan. Tel: +81 236285212, Fax: +81 236285215, e-mail: ckitanak@med.id.yamagata-u.ac.jp (C.K.), m-okada@med.id.yamagata-u.ac.jp (M.O.)

Key Words: Drug repositioning, repurposing, apoptosis, drug resistance. inevitable in the majority of cases after seemingly successful initial treatment, accounting for the poor prognosis of ovarian cancer (3). A growing body of evidence now suggests that cancer stem cells (CSCs), a rare subpopulation of highly chemoresistant tumor cells, play a key role in such post-treatment recurrence of ovarian cancer, and elimination of the CSC population is therefore expected to be a promising approach to improving the survival of patients with ovarian cancer (4-6). However, currently, much remains unclear about the molecular mechanisms underlying the chemoresistance of ovarian CSCs, rendering it difficult to develop methods by which to target such mechanisms.

CEP-1347 is a small-molecule inhibitor of mixed lineage kinases (MLKs), whose safety in humans but not efficacy per se was demonstrated in a large-scale phase II/III clinical trial in early Parkinson's disease (7). In the field of oncology, we recently revealed that CEP-1347 has an anti-CSC effect, by demonstrating that it promotes the differentiation of CSCs derived from a number of cancer types, including ovarian CSCs, in vitro and that systemic administration of CEP-1347 eliminates CSCs in tumors in vivo (8). However, the possible effect of CEP-1347 on the chemoresistance of CSCs is yet to be explored.

In this study, we investigated the mechanism underlying the chemoresistance of ovarian CSCs and examined whether CEP-1347 could chemosensitize ovarian CSCs by disrupting the mechanism.

\section{Materials and Methods}

Antibodies and reagents. Antibodies against sex determining region Y-box 2 (SOX2, \#3579), Survivin (\#2808), glyceraldehyde 3phosphate dehydrogenase (GAPDH, \#5174), mixed lineage kinase 3 (MLK3, \#2817), and cleaved caspase-3 (Asp175) (\#9661) were purchased from Cell Signaling Technology, Inc. (Danvers, MA, USA). Anti-E-cadherin (sc-8426) was from Santa Cruz Biotechnology, Inc. (Santa Cruz, CA, USA). CEP-1347 and 
paclitaxel were purchased from TOCRIS Bioscience (Bristol, UK) and Sigma (St. Luis, MO, USA), respectively, and were dissolved in dimethylsulfoxide (DMSO) to prepare $1 \mathrm{mM}$ stock solutions.

Cell culture. Ovarian CSC lines A2780 CSLC and TOV21G CSLC used in this study were established from human ovarian cancer cell lines A2780 and TOV21G, respectively, and were maintained under the monolayer stem cell culture condition (8-11). In brief, cells were cultured on collagen I-coated dishes (IWAKI, Tokyo, Japan) in stem cell culture medium [Dulbecco's modified Eagle's medium (DMEM)/F12 medium supplemented with 1\% B27 (Thermo Fisher Scientific, Waltham, MA, USA), $20 \mathrm{ng} / \mathrm{ml}$ epidermal growth factor (EGF) and fibroblast growth factor 2 (FGF2) (Peprotech Inc., Rocky Hill, NJ, USA), D-(+)-glucose (final concentration=26.2 mM), Lglutamine (final concentration $=4.5 \mathrm{mM}$ ), 100 units $/ \mathrm{ml}$ penicillin, and $100 \mu \mathrm{g} / \mathrm{ml}$ streptomycin]. Stem cell culture medium was changed approximately every 3 days, and EGF and FGF2 were added to the culture medium every day. Differentiation of ovarian CSCs was induced by culturing the cells under a differentiation-inducing condition (DMEM/F12 containing 10\% fetal bovine serum, 100 units $/ \mathrm{ml}$ penicillin, and $100 \mu \mathrm{g} / \mathrm{ml}$ streptomycin) for 5 days. The authenticity of the cells was verified by genotyping of short tandem repeat (STR) loci (Bio-Synthesis Inc., Lewisville, TX, USA) followed by comparison to the American Type Culture Collection STR database (http://www.atcc.org/STR_Database.aspx) for Human Cell Lines.

Immunoblot analysis. Immunoblot analysis was conducted as described previously $(8,9,12)$. Drug-treated ovarian CSCs were washed with ice-cold phosphate-buffered saline and lysed in RIPA buffer [10 mM Tris/ $\mathrm{HCl}$ (pH 7.4), $0.1 \%$ sodium dodecyl sulfate (SDS), $0.1 \%$ sodium deoxycholate, $1 \% \mathrm{NP}-40,150 \mathrm{mM} \mathrm{NaCl}, 1 \mathrm{mM}$ EDTA, $1.5 \mathrm{mM} \mathrm{Na}_{3} \mathrm{VO}_{4}, 10 \mathrm{mM} \mathrm{NaF}, 10 \mathrm{mM}$ sodium pyrophosphate, $10 \mathrm{mM}$ sodium $\beta$-glycerophosphate and $1 \%$ protease inhibitor cocktail set III (Wako Pure Chemical Industries, Ltd, Osaka, Japan)]. After centrifugation for $10 \mathrm{~min}$ at $14,000 \times g$ at $4{ }^{\circ} \mathrm{C}$, the supernatants were recovered as cell lysates. When solubilization of membrane proteins including E-cadherin was required to detect expression of stem cell and differentiation markers, self-renewing and differentiated ovarian CSCs were lysed in RIPA buffer, followed by immediate addition of the same volume of $2 \times$ Laemmli buffer $(125 \mathrm{mM}$ Tris/ $\mathrm{HCl}$ [pH 6.8], $4 \%$ SDS, $10 \%$ glycerol) and boiling at $95^{\circ} \mathrm{C}$ for $10 \mathrm{~min}$. The protein concentration was determined using a BCA protein assay kit (Pierce Biotechnology, Inc., Rockford, IL, USA). Samples containing equal amounts of protein were resolved by SDS-polyacrylamide gel electrophoresis and transferred to polyvinylidene fluoride membranes. The membranes were probed with a primary antibody against SOX2, E-cadherin, survivin, MLK3, GAPDH or cleaved caspase-3, and subsequently with a horseradish peroxidase (HRP)-conjugated secondary antibody as recommended by the manufacturer of each antibody. Specific bands were visualized using Immobilon Western Chemiluminescent HRP Substrate (Merck Millipore, Billerica, MA, USA) and detected semi-quantitatively by a ChemiDoc Touch (BioRad, Hercules, CA, USA).

Gene silencing by siRNA. siRNAs against human survivin (BIRC5; \#1 HSS HSS 179403, and \#2 HSS179404) and Medium GC Duplex \#2 of Stealth RNAi ${ }^{\mathrm{TM}}$ siRNA Negative Control Duplexes (as a non-targeting control) were purchased from Thermo Fisher Scientific. Briefly, A2780 CSLC and TOV21G CSLC cells were transiently transfected with siRNAs against survivin (siSur) or with a control siRNA and were

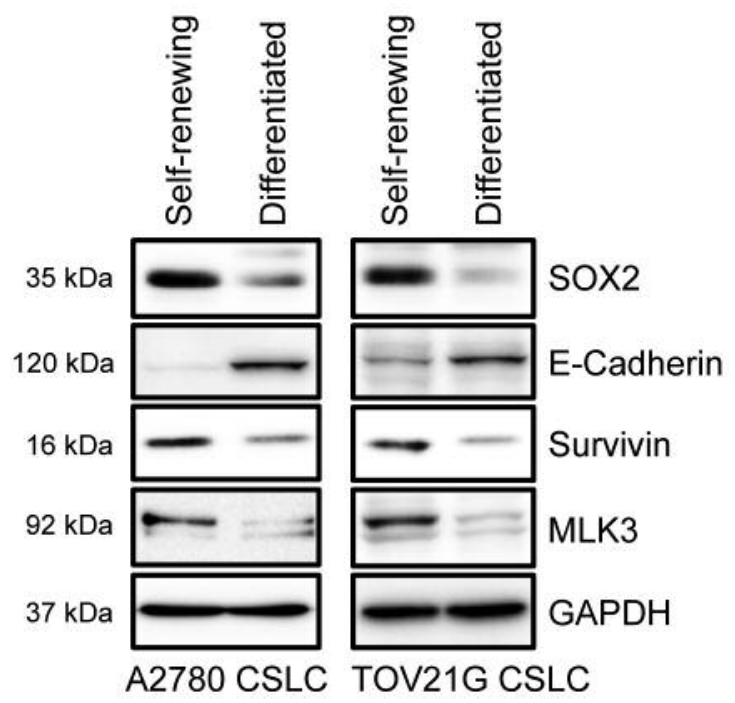

Figure 1. Differential expression of survivin in ovarian cancer stem cells (CSCs) and in their differentiated counterparts. The expression of sex determining region Y-box 2 (SOX2), E-cadherin, survivin, mixed lineage kinase 3 (MLK3), and glyceraldehyde 3-phosphate dehydrogenase $(G A P D H)$ in self-renewing (maintained under the stem cell culture condition) and differentiated (cultured under the differentiation-inducing condition) ovarian CSC lines, A2780 CSLC and TOV21G CSLC, was analyzed by immunoblotting. Note that the expression of SOX2, a stem cell marker, was increased in self-renewing cells, whereas that of $E$ cadherin, a marker of differentiation, was increased in differentiated cells.

treated on the next day without or with $2 \mathrm{nM}$ paclitaxel for 3 days. The cells were then subjected to immunoblot analysis of survivin and to cell death assay using propidium iodide (PI). Transfection of siRNAs was performed using Lipofectamine RNAiMAX (Thermo Fisher Scientific) according to the manufacturer's instruction.

Cell death assay. Cell death assay was performed as previously described $(13,14)$. Briefly, cells were incubated in situ with PI $(1 \mu \mathrm{g} / \mathrm{ml})$ and Hoechst $33342(10 \mu \mathrm{g} / \mathrm{ml})$ for $5 \mathrm{~min}$ at $37^{\circ} \mathrm{C}$ in a $\mathrm{CO}_{2}$ incubator to stain dead cells and cell nuclei, respectively. Then the numbers of PI-and Hoechst-positive cells were scored under a fluorescence microscope (CKX41; OLYMPUS, Tokyo, Japan), and the percentage of PI-positive cells (dead cells) relative to Hoechst-positive cells (total cells) was determined. Cell death assays were performed in triplicate.

Cell viability assay. Cell viability assay was conducted as described previously $(15,16)$. Briefly, cell viability was determined by tetrazolium salt reduction method using WST-8 (Cell Counting Kit8; Dojindo Laboratories, Kumamoto, Japan) according to the manufacture's instruction. Cells (500/well) plated in 96-well collagen I-coated plates were treated or not on the next day with $2 \mathrm{nM}$ paclitaxel in the absence or presence of $200 \mathrm{nM}$ CEP-1347 for 3 days, followed by culture in the absence of any drugs for another 4 days. WST- 8 reagent was then added and the cells were incubated for $1-3 \mathrm{~h}$ at $37^{\circ} \mathrm{C}$. Absorbance at $450 \mathrm{~nm}$ was measured using a microplate reader (Model 680; Bio-Rad). Relative cell viability was 

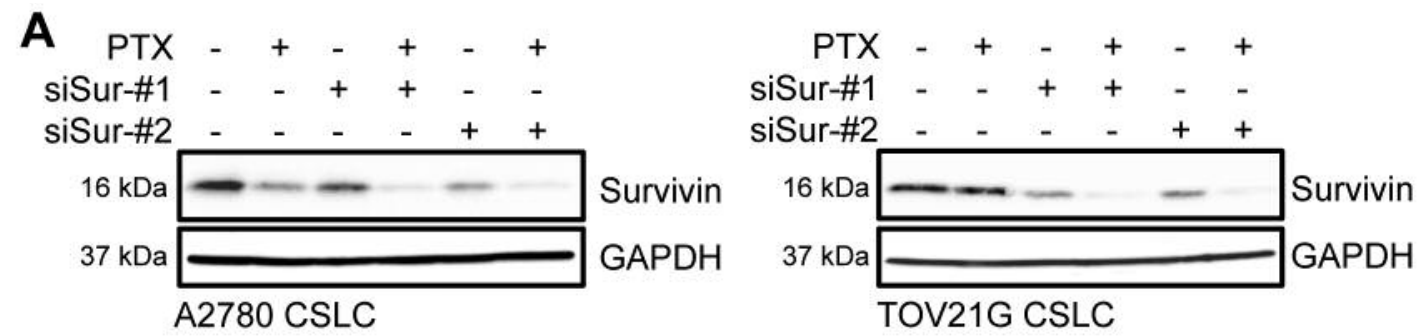

B
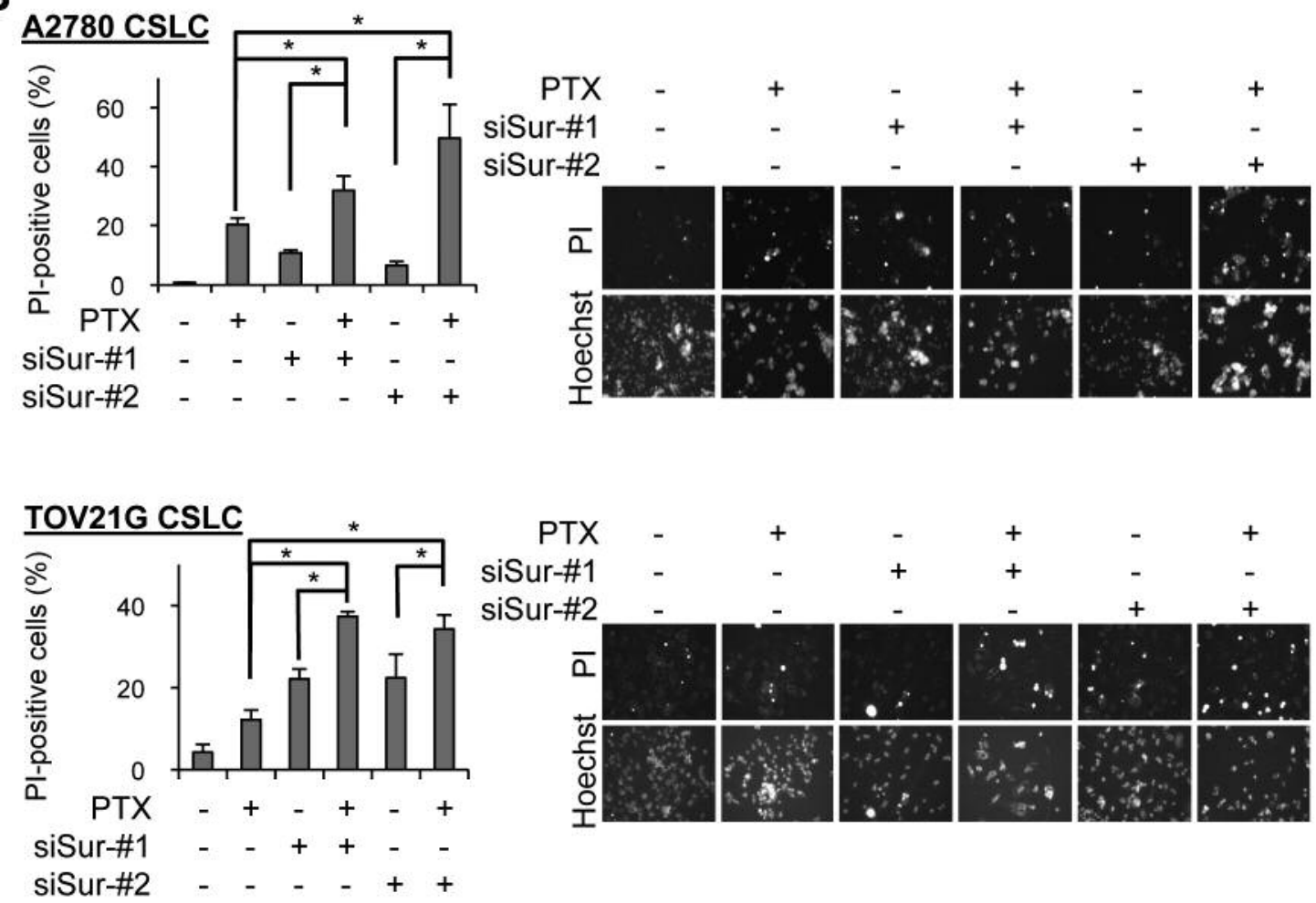

Figure 2. Role of survivin expression in the paclitaxel resistance of ovarian cancer stem cells. A2780 CSLC and TOV21G CSLC cells were transfected with siRNAs against survivin (siSur\#1 or siSur\#2) or with a control RNA. The cells were then treated, starting the next day, without or with paclitaxel (PTX, 2 nM) for 3 days and then subjected to immunoblot analysis of survivin (A) or to cell death assay using propidium iodide (PI) as a vital dye $(B)$. In $(B)$, values represent means $+S D$ from triplicate samples of a representative experiment repeated with similar results. Representative fluorescent images of PI-positive (upper rows) and Hoechst-positive (lower rows) cells are shown on the right. GAPDH: Glyceraldehyde 3-phosphate dehydrogenase. *Significantly different at $p<0.05$.

calculated as a percentage of absorbance of treated samples relative to that of controls. Cell viability assays were performed in triplicate.

Statistical analysis. Results are expressed as the mean and standard deviation (SD), and differences were compared using the two-tailed Student's $t$-test. $p$-Values of less than 0.05 were considered statistically significant.

\section{Results}

Increased survivin expression is involved in paclitaxel resistance of ovarian CSCs. As an approach to exploring the mechanisms involved in the chemoresistance of ovarian CSCs, we searched for chemoresistance-related proteins that are differentially expressed between ovarian CSCs and their differentiated counterparts. The results of western blot analysis using A2780 CSLC and TOV21G CSLC, ovarian CSC lines derived from ovarian cancer cell lines A2780 and TOV21G, respectively, indicated that among chemoresistance-related proteins, survivin was overexpressed in both A2780 CSLC and TOV21G CSLC cells as compared with their respective non-CSC counterparts that had undergone differentiation in a differentiation-inducing culture condition (Figure 1). 
Since survivin is an anti-apoptotic protein implicated in chemoresistance of ovarian cancer cells (17-20), we next wished to determine the impact of survivin expression in these ovarian CSC lines on their sensitivity to paclitaxel, a first-line chemotherapeutic agent in the treatment of ovarian cancer (2). To this end, two independent siRNAs against survivin were introduced to knockdown survivin expression in A2780 CSLC and TOV21G CSLC cells (Figure 2A). Notably, even in the absence of paclitaxel, the proportion of dead cells somewhat increased in survivin-knockdown cells as compared with control-knockdown cells (Figure 2B), suggesting that the basal level of survivin expression in ovarian CSCs may have a role in the maintenance of their survival to some extent. We then went on to examine the impact of survivin-knockdown on paclitaxel sensitivity of ovarian CSCs. Whereas treatment of control-knockdown cells with $2 \mathrm{nM}$ paclitaxel caused only marginal or modest increase in the proportion of dead cells, cell death was significantly promoted in cells in which survivin expression was knocked down using either of the siRNAs against survivin (Figure 2B). These results suggest that the increased survivin expression in ovarian CSCs is critically involved in their paclitaxel resistance.

CEP-1347 reduces survivin expression and sensitizes ovarian CSCs to paclitaxel. Given that survivin expression is involved in paclitaxel resistance of ovarian CSCs, we next wished to identify drugs with which to therapeutically target survivin expression in order to overcome paclitaxel resistance associated with survivin overexpression. Since a parallel search for signaling molecules differentially expressed in ovarian CSCs and their differentiated counterparts revealed that the expression levels of MLK3 closely paralleled those of survivin in both A2780 CSLC and TOV21G CSLC cell lines (Figure 1), we surmised that MLK3 might have a role in survivin expression and therefore utilized CEP-1347, a small-molecule MLK inhibitor with a known safety profile in humans. The results indicated that CEP-1347 efficiently inhibited survivin expression in both ovarian CSC lines (Figure 3). Importantly, CEP-1347 reduced survivin expression in a concentration-dependent manner at $300 \mathrm{nM}$ or below, i.e. at concentrations that have been shown to be clinically relevant in human studies $(21,22)$ (Figure 3A). Time-course analysis demonstrated that survivin expression in cells treated with CEP-1347 at $200 \mathrm{nM}$ decreased over the course of 3 days (Figure 3B).

Having demonstrated the ability of CEP-1347 to inhibit survivin expression, we next asked whether CEP-1347 sensitizes ovarian CSCs to paclitaxel. To examine this, the ovarian CSC lines were treated with paclitaxel in the presence and absence of CEP-1347. Treatment with CEP1347 alone at $200 \mathrm{nM}$ modestly induced cell death in both ovarian CSC lines, which may be in line with our earlier
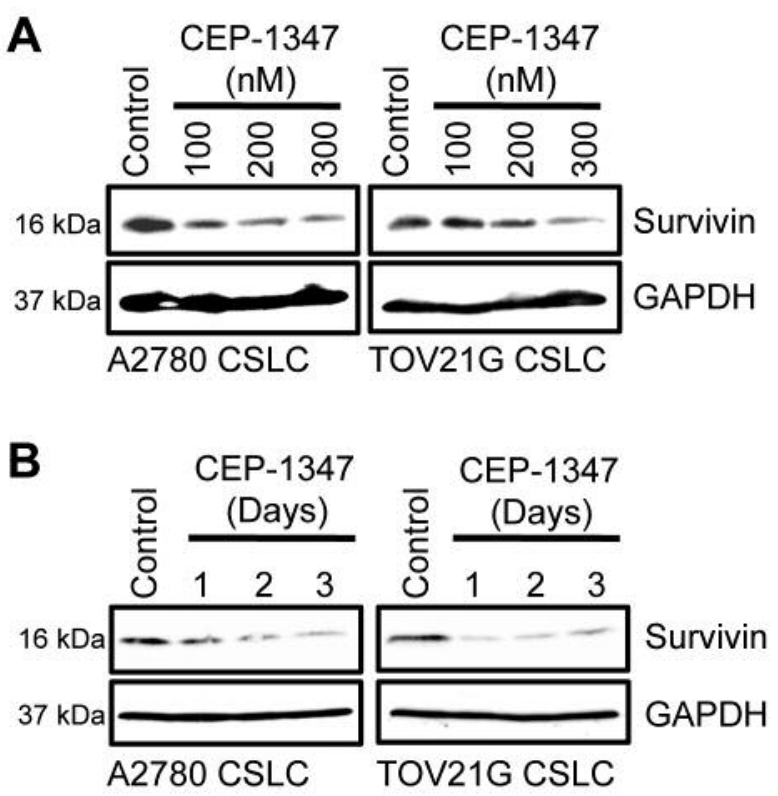

Figure 3. The effect of CEP-1347 on survivin expression in ovarian cancer stem cells. A2780 CSLC and TOV21G CSLC cells were treated with CEP-1347 at the indicated concentrations for 3 days (A) or at $200 \mathrm{nM}$ for the indicated times (B). Control cells were treated without CEP-1347 for 3 days. The cells were subjected to immunoblot analysis of survivin expression. GAPDH: Glyceraldehyde 3-phosphate dehydrogenase.

observation that knockdown of survivin alone was sufficient to reduce their viability (Figure 4A). Treatment of the cells with paclitaxel alone at $2 \mathrm{nM}$ also caused marginal levels of cell death (Figure 4A) as shown earlier (Figure 2B). However, strikingly, treatment of the ovarian CSC lines with the combination of CEP-1347 and paclitaxel resulted in a significant increase in the proportion of dead cells (Figure 4A), in support of the idea that CEP-1347 sensitized the cells to paclitaxel. Further analysis showed that this cell death was accompanied by caspase activation, as indicated by the increased expression of apoptosis-specific cleaved caspase 3 (Figure 4B), suggesting that the caspase-dependent apoptotic program may be involved in cell death induced by CEP- 1347 in the presence and absence of paclitaxel.

Finally, in order to exclude the possibility that CEP-1347 was simply advancing the time-kinetics of cell death that would otherwise eventually occur, we determined whether concurrent treatment with CEP-1347 and paclitaxel for a defined period leads not only to short-term increase in cell death, but also to sustained inhibition of cellular growth, which may be of significance also from a therapeutic point of view. When ovarian CSCs treated with a combination of drugs for 3 days were then allowed to grow in the absence of the drugs, growth was inhibited more efficiently by the combination of CEP-1347 and paclitaxel than either 


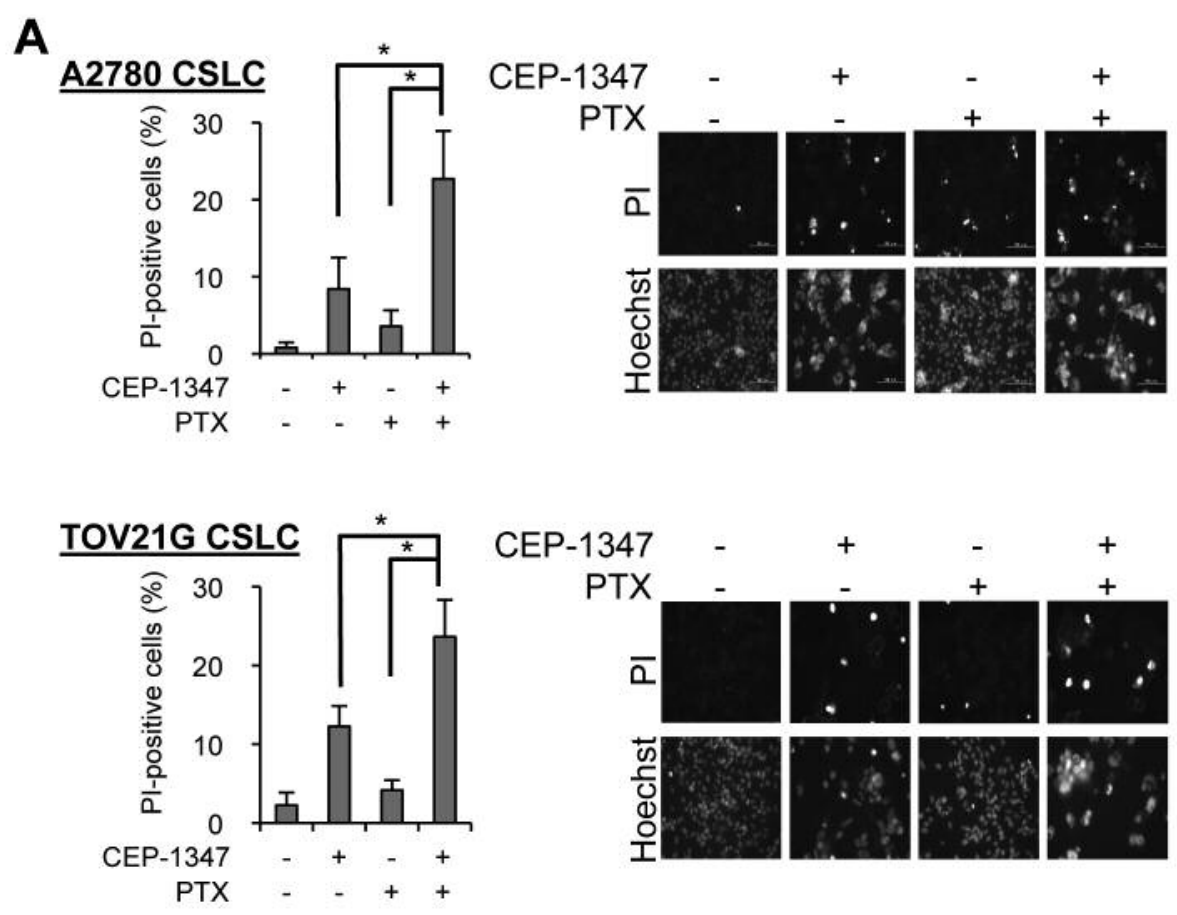

\section{B}
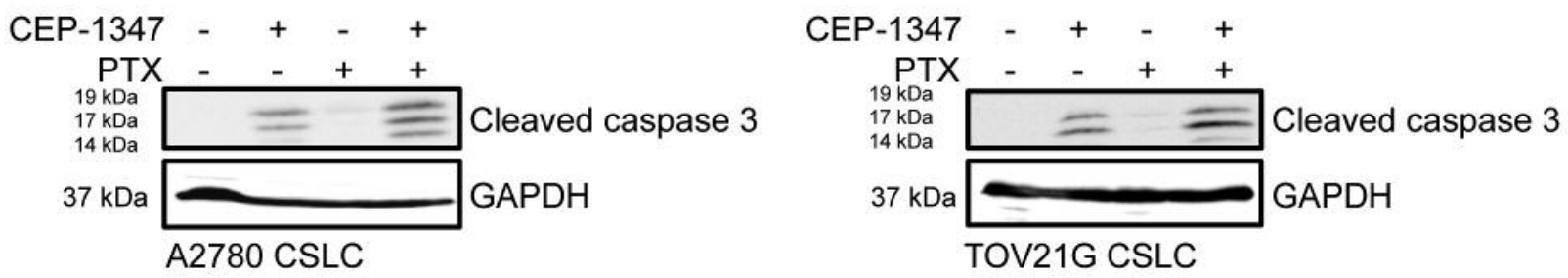

Figure 4. CEP-1347 promotes paclitaxel-induced apoptosis in ovarian cancer stem cells. A: A2780 CSLC and TOV21G CSLC cells treated without or with paclitaxel (PTX, $2 \mathrm{nM})$ in the absence or presence of CEP-1347 (200 nM) for 3 days were subjected to cell death assay using propidium iodide (PI). Left: Values represent means $+S D$ from triplicate samples of a representative experiment repeated with similar results. Right, representative fluorescent images of PI-positive (upper rows) and Hoechst-positive (lower rows) cells are shown. B: Cells treated as described above were subjected to immunoblot analysis of cleaved caspase 3. GAPDH: Glyceraldehyde 3-phosphate dehydrogenase. *Significantly different at $p<0.05$.

treatment alone (Figure 5). These results suggest that the combinatorial use of CEP-1347 and paclitaxel may be beneficial in achieving net reduction in the population of ovarian CSCs.

\section{Discussion}

CSCs are now considered primarily responsible for posttreatment tumor recurrence and hence for the poor prognosis of patients with ovarian cancer (4-6). Overcoming the chemoresistance of ovarian CSCs is, therefore, expected to contribute to improved survival in patients with ovarian cancer, but the underlying mechanisms of chemoresistance are mostly unknown. Here in this study, we showed that chemoresistance-related molecule survivin was overexpressed in A2780 CSLC and TOV21G CSLC cells as compared with their respective differentiated counterparts. This finding is in line with a previous observation that survivin was overexpressed in cancer stem-like cells derived from a human ovarian cancer cell line SKOV3 as compared with their differentiated counterparts (23). Of note, while it was shown in that study that exogenous overexpression of survivin conferred resistance to paclitaxel and cisplatin in otherwise chemosensitive SKOV3 cells, it remained to be shown whether the endogenous expression of survivin in stem-like SKOV3 cells played a key role in their chemoresistance. In this regard, in the present study, we have clearly demonstrated through knockdown experiments that 

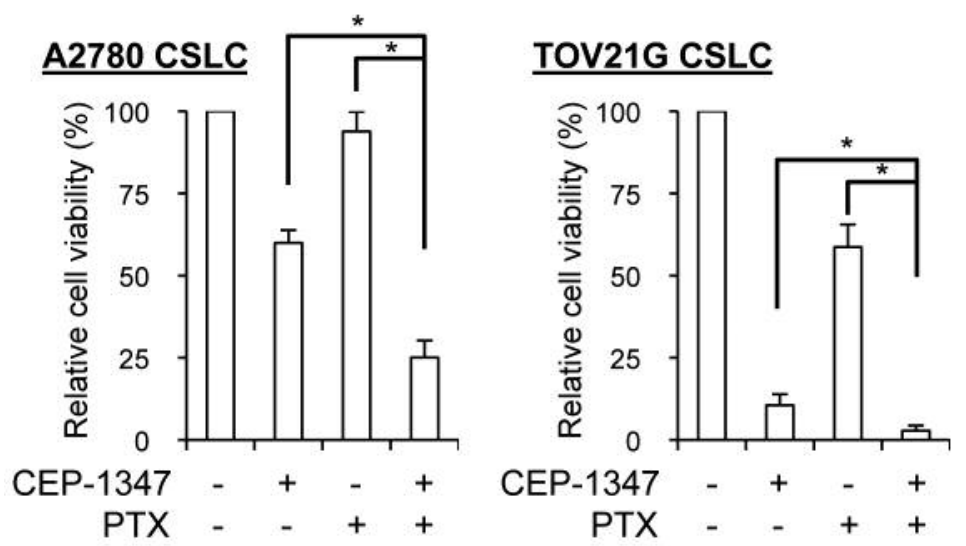

Figure 5. Sustained growth-inhibitory effect of CEP-1347 combined with paclitaxel on ovarian cancer stem cells. A2780 CSLC and TOV21G CSLC cells treated without or with paclitaxel (PTX, $2 \mathrm{nM})$ in the absence or presence of CEP-1347 (200 nM) for 3 days were cultured for another 4 days in the absence of any drug. The cells were then subjected to cell viability assay using WST-8. Values represent means $+S D$ from triplicate samples of a representative experiment repeated with similar results. GAPDH: Glyceraldehyde 3-phosphate dehydrogenase. ${ }^{*}$ Significantly different at $p<0.05$.

the endogenous expression of survivin in ovarian CSCs had an essential role in their chemoresistance. Thus, our findings combined with those of the earlier study suggest that survivin overexpression may be a key factor in the mechanism of chemoresistance of ovarian CSCs.

Survivin is an anti-apoptotic protein that inhibits the core machinery of the intrinsic/mitochondrial apoptotic pathway and as such contributes in a variety of cell types to cellular resistance to a wide range of chemotherapeutic agents acting in an apoptosis-dependent manner (24, 25). Importantly, survivin is selectively overexpressed in cancer cells and not in terminally differentiated adult tissues, which makes it an attractive target of therapeutic drugs to be delivered systemically (24).

Given our current demonstration of the critical role of survivin overexpression in conferring chemoresistance on ovarian CSCs, we expected that drugs targeting survivin would sensitize ovarian CSCs to chemotherapy and thus offer an effective strategy to overcome chemoresistance and improve survival in patients with ovarian cancer. We therefore sought to identify in this study such survivintargeting agents and successfully discovered CEP-1347 as a candidate. CEP-1347 is a small-molecule inhibitor of MLKs that was originally synthesized as a derivative of the natural product K-252a found in broth of Narcodiopsis bacterium (26). The molecular targets of CEP-1347 were unknown at the time of its discovery, but MLKs, upstream regulators of c-Jun $N$-terminal kinase (JNK), were later identified as its targets (27), subsequent to the discovery that CEP-1347 is an inhibitor of the JNK signaling pathway (28). Following the demonstration of the roles of the MLK-JNK pathway in Parkinson's disease and HIV neurotoxicity in preclinical in vitro and in vivo studies $(29,30), \mathrm{CEP}-1347$ was advanced to human clinical trials for Parkinson's disease and HIVassociated encephalitis $(7,31)$, which showed that the dosages of CEP-1347 tested in the trials were well tolerated, with plasma concentrations of CEP-1347 reaching $~ 300 \mathrm{nM}$ (21) and $700 \mathrm{nM}$ (22). Although the efficacy of CEP-1347 in Parkinson's disease and HIV-associated encephalitis is yet to be successfully demonstrated, such pharmacokinetic data from these trials have provided valuable information on the clinically relevant concentration range of CEP-1347. Importantly, the concentration of CEP-1347 we used in this study to inhibit survivin expression and chemosensitize ovarian CSCs was $200 \mathrm{nM}$, which falls well within the above range of clinically relevant concentrations. The results of this study therefore suggest use of CEP-1347 may be a viable and feasible therapeutic approach to targeting ovarian CSCs and as such, this study makes a strong case for investigating the potential of this drug to eliminate ovarian CSCs in vivo and to prevent recurrence in preclinical animal models of ovarian cancer. Our results also suggest that MLK3, and possibly the downstream JNK pathway, may be involved in the regulation of survivin expression. However, CEP-1347 is known to have multiple targets besides MLK3, including other MLK family members (27), and we are yet to demonstrate the involvement of MLK3 and the JNK pathway in a conclusive manner. Future elucidation of the detailed mechanism of survivin regulation, including the possible role of MLK3 and the JNK pathway, is expected to provide useful information with which to further identify novel therapeutic targets.

In conclusion, we provided evidence in support of the idea that survivin overexpression may be among the major mechanisms of increased chemoresistance of ovarian CSCs. Use of CEP-1347, which was demonstrated in this study to 
inhibit survivin expression and chemosensitize ovarian CSCs effectively at a clinically relevant concentration, may therefore become a rational and promising strategy to enhance the efficacy of conventional chemotherapy and thereby improve the survival of patients with ovarian cancer.

\section{Conflicts of Interest}

The Authors declare no conflict of interest in regard to this study.

\section{Acknowledgements}

This work was supported by Grants-in-Aid for Scientific Research, for Challenging Exploratory Research, and for Young Scientists from the Ministry of Education, Culture, Sports, Science and Technology of Japan.

\section{References}

1 Torre LA, Bray F, Siegel RL, Ferlay J, Lortet-Tieulent J and Jemal A: Global cancer statistics, 2012. CA Cancer J Clin 65: 87-108, 2015.

2 Hennessy BT, Coleman RL and Markman M: Ovarian cancer. Lancet 374: 1371-1382, 2009

3 Poveda A, Ray-Coquard I, Romero I, Lopez-Guerrero JA and Colombo N: Emerging treatment strategies in recurrent platinum-sensitive ovarian cancer: focus on trabectedin. Cancer Treat Rev 40: 366-375, 2014.

4 Li SS, Ma J and Wong AST: Chemoresistance in ovarian cancer: exploiting cancer stem cell metabolism. J Gynecol Oncol 29: e32, 2018.

5 Walters Haygood CL, Arend RC, Straughn JM and Buchsbaum DJ: Ovarian cancer stem cells: Can targeted therapy lead to improved progression-free survival? World J Stem Cells 6: 441447, 2014

6 Wang X, Li X, Fu X, Bai M, Li X, Mei Q, Nie J, Wu Z and Han W: Eliminating ovarian cancer stem cells: a potential therapeutic target for ovarian cancer chemoresistance. Curr Protein Pept Sci 16: $270-278,2015$.

7 Investigators PSGP: Mixed lineage kinase inhibitor CEP-1347 fails to delay disability in early Parkinson disease. Neurology 69: 1480-1490, 2007.

8 Okada M, Takeda H, Sakaki H, Kuramoto K, Suzuki S, Sanomachi T, Togashi K, Seino S and Kitanaka C: Repositioning CEP-1347, a chemical agent originally developed for the treatment of Parkinson's disease, as an anti-cancer stem cell drug. Oncotarget 8: 94872-94882, 2017.

9 Sakaki H, Okada M, Kuramoto K, Takeda H, Watarai H, Suzuki S, Seino S, Seino M, Ohta T, Nagase S, Kurachi H and Kitanaka C: GSKJ4, a selective jumonji H3K27 demethylase inhibitor, effectively targets ovarian cancer stem cells. Anticancer Res 35: 6607-6614, 2015.

10 Seino M, Okada M, Shibuya K, Seino S, Suzuki S, Ohta T, Kurachi $\mathrm{H}$ and Kitanaka C: Requirement of JNK signaling for self-renewal and tumor-initiating capacity of ovarian cancer stem cells. Anticancer Res 34: 4723-4731, 2014.

11 Seino M, Okada M, Sakaki H, Takeda H, Watarai H, Suzuki S, Seino S, Kuramoto K, Ohta T, Nagase S, Kurachi $H$ and
Kitanaka C: Time-staggered inhibition of JNK effectively sensitizes chemoresistant ovarian cancer cells to cisplatin and paclitaxel. Oncol Rep 35: 593-601, 2016.

12 Kuramoto K, Suzuki S, Sakaki H, Takeda H, Sanomachi T, Seino S, Narita Y, Kayama T, Kitanaka C and Okada M: Licochalcone A specifically induces cell death in glioma stem cells via mitochondrial dysfunction. FEBS Open Bio 7: 835-844, 2017.

13 Okada M, Kuramoto K, Takeda H, Watarai H, Sakaki H, Seino S, Seino M, Suzuki S and Kitanaka C: The novel JNK inhibitor AS602801 inhibits cancer stem cells in vitro and in vivo. Oncotarget 7: 27021-27032, 2016.

14 Takeda H, Okada M, Suzuki S, Kuramoto K, Sakaki H, Watarai $\mathrm{H}$, Sanomachi T, Seino S, Yoshioka T and Kitanaka C: Rhoassociated protein kinase (ROCK) inhibitors inhibit survivin expression and sensitize pancreatic cancer stem cells to gemcitabine. Anticancer Res 36: 6311-6318, 2016.

15 Takeda H, Okada M, Kuramoto K, Suzuki S, Sakaki H, Sanomachi T, Seino S, Yoshioka T, Hirano H, Arita K and Kitanaka C: Antitumor activity of gemcitabine against highgrade meningioma in vitro and in vivo. Oncotarget 8: 9099691008, 2017.

16 Watarai H, Okada M, Kuramoto K, Takeda H, Sakaki H, Suzuki S, Seino S, Oizumi H, Sadahiro M and Kitanaka C: Impact of H3K27 demethylase inhibitor GSKJ4 on NSCLC cells alone and in combination with metformin. Anticancer Res 36: 6083-6092, 2016.

17 Chen L, Liang L, Yan X, Liu N, Gong L, Pan S, Lin F, Zhang $\mathrm{Q}$, Zhao $\mathrm{H}$ and Zheng F: Survivin status affects prognosis and chemosensitivity in epithelial ovarian cancer. Int J Gynecol Cancer 23: 256-263, 2013.

18 Gasowska-Bodnar A, Bodnar L, Dabek A, Cichowicz M, Jerzak M, Cierniak S, Kozlowski W and Baranowski W: Survivin expression as a prognostic factor in patients with epithelial ovarian cancer or primary peritoneal cancer treated with neoadjuvant chemotherapy. Int J Gynecol Cancer 24: 687-696, 2014.

19 Kar R, Palanichamy JK, Banerjee A, Chattopadhyay P, Jain SK and Singh N: Survivin siRNA increases sensitivity of primary cultures of ovarian cancer cells to paclitaxel. Clin Transl Oncol 17: 737-742, 2015.

20 Salzano G, Navarro G, Trivedi MS, De Rosa G and Torchilin VP: Multifunctional polymeric micelles co-loaded with antisurvivin siRNA and paclitaxel overcome drug resistance in an animal model of ovarian cancer. Mol Cancer Ther 14: 10751084, 2015.

21 Group. PS: The safety and tolerability of a mixed lineage kinase inhibitor (CEP-1347) in PD. Neurology 62: 330-332, 2004.

22 Ma Q, Gelbard HA, Maggirwar SB, Dewhurst S, Gendelman HE, Peterson DR, DiFrancesco R, Hochreiter JS, Morse GD and Schifitto G: Pharmacokinetic interactions of CEP-1347 and atazanavir in HIV-infected patients. J Neurovirol 19: 254-260, 2013.

23 Dong Z, Yang L and Lai D: KLF5 strengthens drug resistance of ovarian cancer stem-like cells by regulating survivin expression. Cell Prolif 46: 425-435, 2013.

24 Garg H, Suri P, Gupta JC, Talwar GP and Dubey S: Survivin: A unique target for tumor therapy. Cancer Cell Int 16: 49, 2016.

25 Singh N, Krishnakumar S, Kanwar RK, Cheung $\mathrm{CH}$ and Kanwar JR: Clinical aspects for survivin: A crucial molecule for targeting drug-resistant cancers. Drug Discov Today 20: 578-587, 2015. 
26 Kaneko M, Saito Y, Saito H, Matsumoto T, Matsuda Y, Vaught JL, Dionne CA, Angeles TS, Glicksman MA, Neff NT, Rotella DP, Kauer JC, Mallamo JP, Hudkins RL and Murakata C: Neurotrophic 3,9-bis[(alkylthio)methyl]-and-bis(alkoxymethyl)K-252a derivatives. J Med Chem 40: 1863-1869, 1997.

27 Maroney AC, Finn JP, Connors TJ, Durkin JT, Angeles T, Gessner G, Xu Z, Meyer SL, Savage MJ, Greene LA, Scott RW and Vaught JL: CEP-1347 (KT7515), a semisynthetic inhibitor of the mixed lineage kinase family. J Biol Chem 276: 2530225308, 2001.

28 Maroney AC, Glicksman MA, Basma AN, Walton KM, Knight E, Jr., Murphy CA, Bartlett BA, Finn JP, Angeles T, Matsuda Y, Neff NT and Dionne CA: Motoneuron apoptosis is blocked by CEP-1347 (KT 7515), a novel inhibitor of the JNK signaling pathway. J Neurosci 18: 104-111, 1998.
29 Eggert D, Dash PK, Gorantla S, Dou H, Schifitto G, Maggirwar SB, Dewhurst S, Poluektova L, Gelbard HA and Gendelman HE: Neuroprotective activities of CEP-1347 in models of neuroAIDS. J Immunol 184: 746-756, 2010.

30 Waldmeier P, Bozyczko-Coyne D, Williams M and Vaught JL: Recent clinical failures in Parkinson's disease with apoptosis inhibitors underline the need for a paradigm shift in drug discovery for neurodegenerative diseases. Biochem Pharmacol 72: 1197-1206, 2006.

31 Christophersen J, Robertson P, Hellriegel E and Watling M: CEP1347: A new neuroprotective agent. Mov Disord 17: P178, 2002.

Received May 31, 2018

Revised June 19, 2018

Accepted June 20, 2018 